\title{
Q.
QNEEN'S
UNIVERSITY
BELFAST
}

\section{A Search for Water in a Super-Earth Atmosphere: High-resolution Optical Spectroscopy of 55Cancri e}

Esteves, L. J., de Mooij, E. J. W., Jayawardhana, R., Watson, C., \& de Kok, R. (2017). A Search for Water in a Super-Earth Atmosphere: High-resolution Optical Spectroscopy of 55Cancri e. Astronomical Journal, 153(6), 268. https://doi.org/10.3847/1538-3881/aa7133

Published in:

Astronomical Journal

Document Version:

Publisher's PDF, also known as Version of record

Queen's University Belfast - Research Portal:

Link to publication record in Queen's University Belfast Research Portal

Publisher rights

(C) 2017 The American Astronomical Society. All rights reserved.

This work is made available online in accordance with the publisher's policies. Please refer to any applicable terms of use of the publisher

\section{General rights}

Copyright for the publications made accessible via the Queen's University Belfast Research Portal is retained by the author(s) and / or other copyright owners and it is a condition of accessing these publications that users recognise and abide by the legal requirements associated with these rights.

Take down policy

The Research Portal is Queen's institutional repository that provides access to Queen's research output. Every effort has been made to ensure that content in the Research Portal does not infringe any person's rights, or applicable UK laws. If you discover content in the Research Portal that you believe breaches copyright or violates any law, please contact openaccess@qub.ac.uk. 


\title{
A Search for Water in a Super-Earth Atmosphere: High-resolution Optical Spectroscopy of 55Cancri e
}

\author{
Lisa J. Esteves ${ }^{1}$, Ernst J. W. de Mooij ${ }^{2,3}$, Ray Jayawardhana ${ }^{4}$, Chris Watson ${ }^{2}$, and Remco de Kok ${ }^{5,6}$ \\ ${ }^{1}$ Astronomy \& Astrophysics, University of Toronto, 50 St. George Street, Toronto, Ontario M5S 3H4, Canada; esteves@astro.utoronto.ca \\ ${ }^{2}$ Astrophysics Research Centre, School of Mathematics and Physics, Queens University, Belfast, UK; ernst.demooij@dcu.ie, c.a.watson@qub.ac.uk \\ ${ }^{3}$ School of Physical Sciences, Dublin City University, Dublin 9, Ireland \\ ${ }^{4}$ Physics \& Astronomy, York University, Toronto, Ontario L3T 3R1, Canada; rayjay @yorku.ca \\ ${ }^{5}$ Leiden Observatory, Leiden University, Postbus 9513, 2300 RA, Leiden, The Netherlands; r.j.de.kok@sron.nl \\ ${ }^{6}$ SRON Netherlands Institute for Space Research, Sorbonnelaan 2, 3584 CA Utrecht, The Netherlands \\ Received 2016 October 21; revised 2017 May 2; accepted 2017 May 3; published 2017 May 31
}

\begin{abstract}
We present the analysis of high-resolution optical spectra of four transits of 55Cnc e, a low-density super-Earth that orbits a nearby Sun-like star in under $18 \mathrm{hr}$. The inferred bulk density of the planet implies a substantial envelope, which, according to mass-radius relationships, could be either a low-mass extended or a high-mass compact atmosphere. Our observations investigate the latter scenario, with water as the dominant species. We take advantage of the Doppler cross-correlation technique, high-spectral resolution, and the large wavelength coverage of our observations to search for the signature of thousands of optical water absorption lines. Using our observations with HDS on the Subaru telescope and ESPaDOnS on the Canada-France-Hawaii Telescope, we are able to place a $3 \sigma$ lower limit of $10 \mathrm{~g} \mathrm{~mol}^{-1}$ on the mean-molecular weight of $55 \mathrm{Cnc}$ e's water-rich (volume mixing ratio $>10 \%$ ), optically thin atmosphere, which corresponds to an atmospheric scale-height of $\sim 80 \mathrm{~km}$. Our study marks the first high-spectral resolution search for water in a super-Earth atmosphere, and demonstrates that it is possible to recover known water-vapor absorption signals in a nearby super-Earth atmosphere, using highresolution transit spectroscopy with current ground-based instruments.
\end{abstract}

Key words: planets and satellites: atmospheres - planets and satellites: terrestrial planets

\section{Introduction}

In recent years, enormous progress has been made in our understanding of the atmospheric properties of hot Jupiters through observations of transiting extrasolar planets. Notable discoveries include the first detection of sodium (Charbonneau et al. 2002), the first detections of a secondary eclipse from space (Charbonneau et al. 2005; Deming et al. 2005) and the ground (de Mooij \& Snellen 2009; Sing and López-Morales 2009), and detailed studies at high spectral resolution of carbon monoxide in day-side emission spectra (Brogi et al. 2012; Rodler et al. 2012). Apart from the first detections, progress in our understanding of the atmospheric properties of hot Jupiters has been made through analyses of transmission and emission spectra (e.g., Swain et al. 2009; Deming et al. 2013; Stevenson et al. 2014; Sing et al. 2016). For super-Earths, on the other hand, the available information on their atmospheric properties is very limited, mainly due to the much higher signal-to-noise ratio required to measure their atmospheric features, as well as the limited number of super-Earths known to orbit bright stars.

One of the most-studied super-Earths is GJ1214b, a very low-density $\left(\rho \sim 0.4 \rho_{\text {Earth }}\right)$ planet orbiting a M5V star. The planet is expected to either have an extended hydrogen-rich envelope, or be water-rich and have a steam atmosphere (Charbonneau et al. 2009). The current observations (e.g., Bean et al. 2010, 2011; Kreidberg et al. 2014) point toward a featureless transmission spectrum, consistent with a thick cloud layer. HD 97658b, another well-studied super-Earth (Knutson et al. 2014), exhibits a featureless transmission spectrum, which suggests an atmosphere covered by thick clouds or composed of a molecular species much heavier than hydrogen. The observations of these two super-Earths were obtained with the $2.4 \mathrm{~m}$ Hubble Space Telescope, which has the advantage of being above the Earth's atmosphere. However, it is limited by observing efficiency, especially for bright targets. Due to the instruments available, it also has limited wavelength coverage and spectral resolution. The largest ground-based telescopes, on the other hand, have much greater collecting areas than Hubble, and also host sophisticated new instruments, including highresolution spectrographs capable of resolving individual absorption lines of molecules such as water. Although the presence of the Earth's atmosphere has a significant negative impact on ground-based optical observations, the fact that the radial velocity of $55 \mathrm{Cnc}$ e, its host star and Earth's atmosphere change very differently throughout the night allows for the use of crosscorrelation with model spectra to distinguish the planetary signal from tellurics and stellar activity.

\subsection{Cnc e}

Of the known super-Earths, $55 \mathrm{Cnc}$ e is the best target for atmospheric studies from the ground. It orbits a nearby G8V star, currently one of the brightest transiting planet hosts, in under $18 \mathrm{hr}$, making it one of the hottest planets known to date. The observations analyzed in this study take advantage of $55 \mathrm{Cnc}$ 's brightness, and the large collecting area available from the ground, to attain the precision needed to detect a water-rich, low-mass, optically thin atmosphere on $55 \mathrm{Cnc}$ e.

$55 \mathrm{Cnc}$ e was initially discovered and characterized by radial-velocity measurements (McArthur et al. 2004; Fischer et al. 2008). Dawson \& Fabrycky (2010) later revised the analysis, yielding a period of $0.736539 \pm 0.000003$ days and a minimum mass of $8.3 \pm 0.3 M_{\oplus}$. Transits were subsequently detected by Winn et al. (2011) using the MOST satellite, and Demory et al. (2011) using the Spitzer Space Telescope. Winn et al. (2011) find 55Cnc e's mass, radius, and mean density to 
be $8.63 \pm 0.35 M_{\oplus}, 2.00 \pm 0.14 R_{\oplus}$, and $5.9 \pm 1.5 \mathrm{~g} \mathrm{~cm}^{-3}$, respectively, whereas Demory et al. (2011) find values of $7.8 \pm 0.5 M_{\oplus}, 2.1 \pm 0.2 R_{\oplus}$ and $4.8 \pm 1.3 \mathrm{~g} \mathrm{~cm}^{-3}$, respectively. Follow-up transit observations made from space by Gillon et al. (2012), Dragomir et al. (2014), and Demory et al. (2016a), as well as from the ground by de Mooij et al. (2014), report planet radius measurements in agreement with these studies. Altogether, their results suggest that, because $55 \mathrm{Cnc}$ e's mean density is comparable to that of Earth, despite the greater mass (and consequently greater compression), the solid interior must be supplemented by a significant mass of water, gas, or other light elements.

Although a purely silicate interior with no envelope was ruled out (Demory et al. 2011; Winn et al. 2011; Gillon et al. 2012), Madhusudhan et al. (2012) suggest that a carbonrich interior with no envelope could explain 55Cnc e's inflated radius. However, follow-up secondary eclipse, phase-curve, and transit measurements (Demory et al. 2012, 2016a, 2016b; Ridden-Harper et al. 2016; Tsiaras et al. 2016), in conjunction with theoretical models (Lammer et al. 2013; Ito et al. 2015; Kite et al. 2016), point to the existence of an envelope.

Mass-radius relationships for planets with envelopes composed of either hydrogen-helium or water were presented in Winn et al. (2011), Demory et al. (2011), and Gillon et al. (2012). These studies show that 55Cnc e's density can be explained by either a low-mass, hydrogen-helium or a highmass, water-dominated atmosphere; however, simple atmospheric escape calculations show that the former is unlikely due to their short evaporation timescale, on the order of a million years for hydrogen (Valencia et al. 2010). Assuming the evaporation timescale of water is much longer, on the order of a billion years, they conclude that a water-dominated envelope is more likely. However, Lammer et al. (2013) calculate the theoretical atmospheric mass-loss of a hydrogen-dominated envelope on $55 \mathrm{Cnc} \mathrm{e}$ that is being irradiated by XUV photons (Ehrenreich et al. 2012), and find that thermal massloss rates are $\sim 10$ times lower than those of a typical hotJupiter, such as HD 209458b. As a result, Lammer et al. (2013) conclude that one can expect that $55 \mathrm{Cnc}$ e would not lose its hydrogen envelope during its remaining lifetime.

Demory et al. (2012) report a Spitzer secondary eclipse depth of $131 \pm 28 \mathrm{ppm}$ corresponding to a brightness temperature of $2360 \pm 300 \mathrm{~K}$. Ito et al. (2015) find that this brightness temperature is in agreement with the theoretically predicted eclipse depth created by a mineral atmosphere on $55 \mathrm{Cnc}$ e. Their study investigates the radiative properties of an atmosphere that is in gas/melt equilibrium with the underlying magma ocean. Their equilibrium calculations yielded a mineral atmosphere (i.e., $\mathrm{Na}, \mathrm{K}, \mathrm{Fe}, \mathrm{Si}, \mathrm{SiO}, \mathrm{O}$, and $\mathrm{O}_{2}$ as the major atmospheric species), and radiative absorption line data was used to calculate theoretical eclipse depths.

Demory et al. (2016a, 2016b) observed 55Cnc e's orbital phase curve and eclipse using Spitzer and report highly asymmetric day-side thermal emission, a strong day-night temperature contrast, and a thermal day-side temperature that varies by a factor of 3.7 over a period of two years. These studies hypothesize that phase curve properties are the result of either an optically thick atmosphere with heat recirculation confined to the planetary day-side, or a planet devoid of atmosphere, with low-viscosity magma flowing at the surface. However, Kite et al. (2016) find, using basic models, that heat transport by magma currents is insufficient to explain the antistellar-hemisphere temperature found by Demory et al. (2016b), and therefore an atmosphere is indicated.

The composition and properties of a likely gaseous envelope on $55 \mathrm{Cnc}$ e has also been the focus of recent space-based studies. Tsiaras et al. (2016) analyze Hubble Space Telescope observations and report the detection of features at 1.42 and $1.54 \mu \mathrm{m}$. Using Bayesian spectral retrieval, they conclude that these features are likely the result of trace amounts of $\mathrm{HCN}$, retained within a lightweight atmosphere, presumably composed of mostly hydrogen and helium. Using ground-based data, Ridden-Harper et al. (2016) report a tentative detection of an exosphere via absorption from sodium $(3 \sigma)$ and singly ionized calcium ( $4 \sigma$, variable).

Our study, similar to Ridden-Harper et al. (2016), uses ground-based high-resolution transmission spectra to search for absorption signatures of 55Cnc e's atmosphere. However, we take advantage of the large wavelength coverage and high spectral resolution of our observations to search for the signature of thousands of optical absorption lines produced by water. We note that, although $55 \mathrm{Cnc}$ e has been called a possible water-world, the presence of water is not expected if the planet's C/O abundance is $>1$ ( $\mathrm{Hu} \&$ Seager 2014). However, it is unclear if $55 \mathrm{Cnc}$ e is expected to be carbon-rich, as its host star has been reported as both carbon-rich (Delgado Mena et al. 2010, C/O = 1.12 \pm 0.09 ) and carbon-poor (Teske et al. 2013, $\mathrm{C} / \mathrm{O}=0.78 \pm 0.08)$.

\subsection{High-resolution Cross-correlation of Spectra}

Here, we present the results of our search for water in four high-resolution ground-based transit observations of 55Cnc e. To detect water, we use a Doppler cross-correlation technique that combines the signal from thousands of water lines and uses the difference in Doppler shift to disentangle 55Cnc e's spectra from the atmospheric spectra of its host star and the Earth.

This technique was pioneered by Snellen et al. (2010) to detect carbon monoxide in the atmosphere of HD209458 b during the planet's transit. It has also been successfully used to detect carbon monoxide in the day-side spectra of $\tau$ Bootis $b$ (Brogi et al. 2012; Rodler et al. 2012), as well as carbon monoxide and water in the transmission spectra of $\beta \mathrm{Pic} b$ (Snellen et al. 2014), HD189733 b (Birkby et al. 2013; de Kok et al. 2013; Rodler et al. 2013a; Lockwood et al. 2014; Brogi et al. 2016), HD179949 b (Brogi et al. 2014), and 51Peg b (Brogi et al. 2013). In addition, a similar study in the optical claims to have detected reflected light from 51Peg b (Martins et al. 2015), and a study at ultraviolet wavelengths claims a low-significance detection of reflected light from $\tau$ Bootis $b$ (Rodler et al. 2013b).

We apply this technique to search for water at optical wavelengths in the transmission spectrum of the super-Earth $55 \mathrm{Cnc}$ e. We present our high-resolution observations of four transits in Section 2. In Section 3, we describe the data reduction, which includes extracting and normalizing the 1D spectra, then removing contamination from systematics and stellar and telluric absorption. Our analysis, in Section 4, describes our cross-correlation and retrieval procedure, as well as the atmospheric models used. We present and discuss our results in Section 5. 
Table 1

Summary of Observations

\begin{tabular}{|c|c|c|c|c|c|c|}
\hline Night & $\begin{array}{l}\text { Date } \\
\text { (UT) }\end{array}$ & Instrument/Telescope & $\begin{array}{l}\text { Duration } \\
\quad(\mathrm{hr})\end{array}$ & $\begin{array}{l}\# \text { of } \\
\text { Frames }\end{array}$ & $\begin{array}{l}\text { Cadence } \\
\text { (s) }\end{array}$ & $\begin{array}{l}\text { Average SNR } \\
\text { of Continuum }\end{array}$ \\
\hline 1 & $2014 \mathrm{Feb} 9$ & ESPaDOnS/CFHT & 4 & 76 & 187 & 150 \\
\hline 3 & 2014 Dec 12 & HDS/Subaru & 6 & 136 & 192 & 370 \\
\hline 4 & 2015 Jan 9 & HDS/Subaru & 8.5 & 158 & 192 & 440 \\
\hline
\end{tabular}

\section{Observations}

We observed two transits of 55Cnc e with the Echelle SpectroPolarimetric Device for the Observation of Stars (ESPaDOns; Donati 2003) at the Canada-France Hawai'i Telescope (CFHT). The first of these transits was observed on the night of 2014 February 9 UT (hereafter $\mathrm{N}_{1}$ ) and the second on 2014 April 23 UT (hereafter $\mathrm{N}_{2}$ ). The observations were done in the Queued Service Observing mode, where the observations are executed by the staff at CFHT. For both nights, we used the instrument in the "Star+Sky" mode, resulting in a resolution of $R \sim 68,000$. We used an exposure time of $149 \mathrm{~s}$, resulting in an average cadence of $\sim 187 \mathrm{~s}$. On each night, we observed for just over $4 \mathrm{hr}$ and obtained 76 frames per night, of which 29 were during transit. The weather during $\mathrm{N}_{1}$ was photometric throughout the observations, with a median seeing of 0 ". 5 . During $\mathrm{N}_{2}$, the weather was photometric at the start, with some thin cirrus toward the end of the observations, and seeing varied between $\sim 0$. 7 and $\sim 1$ !' 1 . The average continuum SNR of the spectra were approximately 150 and 140 for $\mathrm{N}_{1}$ and $\mathrm{N}_{2}$, respectively. The extracted CFHT spectra cover a wavelength range of 5060-7950 A, spanning 16 orders.

We also observed two transits with the High Dispersion Spectrograph (HDS; Noguchi et al. 2002) at the Subaru Telescope. We observed one transit on 2014 December 12, (hereafter $\mathrm{N}_{3}$ ) and a second on 2015 January 9 (hereafter $\mathrm{N}_{4}$ ). For both nights, the observations were taken using the \#1 image slicer rather than the slit, to allow for a high signal-tonoise ratio at a spectral resolution of $R \sim 110,000$ (Tajitsu et al. 2012). To reduce the overheads, we binned by two pixels in the spatial direction, leaving the binning in the spectral direction unchanged. We set the exposure to $120 \mathrm{~s}$, resulting in an average cadence of $\sim 192 \mathrm{~s}$. We observed the first transit for just over $6 \mathrm{hr}$ and the second for $8.5 \mathrm{hr}$, during which we obtained 136 and 158 total frames. During each night, 28 frames were taken during transit. The weather during $\mathrm{N}_{3}$ was photometric throughout the observations, with a median seeing of 0.5 . During $\mathrm{N}_{4}$, the weather was also photometric, but the seeing varied between $\sim 0$ ". 4 and $\sim 0$ ". 7 . The average continuum SNR of the spectra were approximately 370 and 440 for $\mathrm{N}_{3}$ and $\mathrm{N}_{4}$, respectively. The extracted Subaru spectra cover a wavelength range of 5240-7890 A, spanning 38 orders. A summary of the observations can be found in Table 1.

\section{Data Reduction and Correction of Systematic Effects}

\subsection{Extraction and Alignment of the 1D Spectra}

The CFHT observations $\left(\mathrm{N}_{1}\right.$ and $\left.\mathrm{N}_{2}\right)$ were reduced and extracted using the Upena pipeline at CFHT, which is based on Libre ESpRIT (Donati et al. 1997). We use the unnormalized but wavelength-corrected spectra produced by the pipeline for the rest of our analysis. Although the pipeline corrects for some drifts in the wavelength solution, we determine any residual shifts directly. To do this, we measure the position of strong telluric lines due to oxygen and water. For $\mathrm{N}_{1}$ and $\mathrm{N}_{2}$, the maximum velocity drift of the telluric lines was less than 0.2 $\mathrm{km} \mathrm{s}^{-1}$, which corresponds to a drift in wavelength of less than $0.0006 \AA$. We subsequently interpolate all frames onto a common wavelength grid in the telluric reference frame, taking these small drifts into account. The extracted CFHT spectra cover a wavelength range of 5060-7950 , spanning 16 orders. The output from this stage, for three orders, can be seen in the top sub-panels of Appendix Figures 4 and 5.

The Subaru observations $\left(\mathrm{N}_{3}\right.$ and $\left.\mathrm{N}_{4}\right)$ were reduced using a combination of IRAF scripts and custom IDL routines. As a first step, the overscan correction was performed for all frames, using the overscan.cl IRAF script ${ }^{7}$ provided by the observatory. Subsequently, the detector nonlinearity (Tajitsu et al. 2012) was corrected using a script provided on the instrument pages. ${ }^{8}$ The individual bias frames were combined in IRAF and subtracted from all remaining frames.

When inspecting the bias-subtracted frames, we identified and removed a low-level crosstalk at the level of $1.4 \times 10^{-3}$, between the two halves of each of the CCDs. The level was determined by varying the crosstalk coefficient until the signal was no longer detectable.

We subsequently combined the individual bias-subtracted flat frames into an uncorrected master flat field using IRAF, and used this to trace the echelle orders. To trace the location of the echelle orders, we determined, at each wavelength, the position of the points on the edge of the echelle order where the flux was $10 \%$ of the peak flux at that wavelength. We then fit a $2 \mathrm{D}$ polynomial to the position of all the echelle orders on an individual $\mathrm{CCD}$, in order to retrieve a global solution. This solution was used for both order masking and spectral extraction.

Subsequently, we removed the scattered light from the master-flat. The scattered light was fit on a wavelength-bywavelength basis by fitting a low-order polynomial to the light between orders for each CCD along the spatial axis, after masking out the orders based on the trace performed earlier. We generated our final, normalized master flat by fitting the flux in the individual orders as a function of wavelength and dividing the unnormalized flat by this fit.

The science frames were subsequently flat-fielded, and we used custom IDL scripts to perform the trace of the spatial centroid of the star in each order, as a function of wavelength. We performed the trace by simultaneously fitting Gaussian profiles to each of the image slices, while fixing the distance between the stellar psf in each of the slices. During the fit, we

\footnotetext{
7 http://www.subarutelescope.org/Observing/Instruments/HDS/hdsql/ overscan.cl

8 http://www.subarutelescope.org/Observing/Instruments/HDS/hdsql/ hdslinear.cl
} 
used a common FWHM for all the slices, but the amplitude for each slice was allowed to vary. As with the flat field, a 2D polynomial was fit to the individual traces in order to obtain a global solution for each CCD.

This trace was used to help identify and correct cosmic rays and bad pixels using a custom IDL script. We determined the average profile in segments with a length of 500 pixels in the spectral direction on an order-by-order basis. These segments were generated in steps of 250 pixels, and the average profile corresponding to the nearest segment to the wavelength under consideration was used. This average profile was scaled to match the local profile, and any pixel discrepant by more than $5 \sigma$ was replaced by the scaled average profile.

After correction for cosmic rays, we removed scattered light in the same way as for the master flat. Finally, we extracted the spectra by summing up the flux over the full spatial extent of each order, thereby directly combining the flux from all five image slices.

Wavelength calibrations were made using ThAr frames taken just before and after the observations of $55 \mathrm{Cnc}$. The positions of the ThAr lines were identified and fitted using the ecidentify routine in IRAF. To maximize observing efficiency, we did not take any ThAr frames during the science observations, but instead relied on the telluric absorption features to track drifts in the wavelength solution. For $\mathrm{N}_{3}$ and $\mathrm{N}_{4}$, the maximum velocity drift of the telluric lines was less than $0.4 \mathrm{~km} \mathrm{~s}^{-1}$, which corresponds to a drift in wavelength of less than $0.002 \AA$. We finally interpolated all spectra within a night onto a common wavelength grid in the telluric reference frame. The extracted Subaru spectra cover a wavelength range of 5240-7890 A, spanning 38 orders. The output from this stage, for three orders, can be seen in the top sub-panels of Appendix Figures 6 and 7.

\subsection{Normalization of Spectra}

Large-scale time-dependent systematics, whose likely cause is the instrument's changing blaze response, were removed, and the spectra were simultaneously normalized. To do this, we first divided an individual spectrum on a night by a nightly reference image, for which we used the first frame of the night. The divided spectrum was then binned by 201 pixels $(\sim 5 \AA)$ for the CFHT data and 41 pixels $(\sim 1 \AA)$ for the Subaru data, fit with a spline and evaluated at the wavelength positions of the unbinned spectrum. The evaluated spline was then divided out of the original spectrum, resulting in the removal of the timevariable blaze response and the normalization of the each spectrum continuum to the reference frame continuum. We then removed the 100 pixels at the edges of each order, in order to avoid contamination from the poor constraints at the edge. We note that, due to the shape of the blaze function, these pixels already have a significantly lower SNR compared to the center of each order, and therefore removing these pixels has minimal impact on the results.

We selected these binsizes in order to avoid removing the absorption lines from the planetary atmosphere, while allowing us to correct the small wavelength scale variations seen in the blaze function for the Subaru observations. This processing results in frames from a single night sharing the same overall blaze function, while maintaining their individual highfrequency signal. The results from the normalization can be seen in the upper middle sub-panels of Appendix Figures 4-7.

\subsection{Removal of Telluric and Stellar Lines}

The stellar and telluric lines were removed using the SYSREM algorithm described by Tamuz et al. (2005). Both in-transit and out-of-transit frames from an individual night were used to determine the correction. Each order was treated separately; for consistency, we chose to apply six iterations of SYSREM to each night of data. We also chose to apply the maximum recommended number of iterations, because the rms of the residuals for several orders does not plateau until six iterations have been applied. After SYSREM was applied, we found the averaged rms for each order, for Nights 1 to 4: 0.004, $0.005,0.002$, and 0.001 , respectively.

This method is able to remove the telluric and stellar absorption features, that are stable in time, while preserving the signal from 55Cnc e's atmosphere. With an average orbital velocity of $228.7 \mathrm{~km} \mathrm{~s}^{-1}$, $55 \mathrm{Cnc}$ e's radial orbital velocity changes rapidly during the transit, from -57.6 to $+57.6 \mathrm{~km} \mathrm{~s}^{-1}$. Our reduction does poorly in the orders with strong, closely spaced telluric lines, particularly between 7600 and $7800 \AA$ where many strong oxygen lines are present. The strong lines make properly modeling the blaze function difficult; as a result, they introduce nonlinear variations that are not removed by SYSREM. Similar to Snellen et al. (2010), before cross-correlating, to reduce contamination from noisy frames, we weighted each of the frames by dividing by their standard deviation. We then similarly weighted each pixel, by dividing by the standard deviation of the pixel's variations throughout the night. This was done to suppress the contribution from noisy frames pixels. The results for this step can be seen in the lower middle sub-panels of Appendix Figures 4-7. A plot of pixel rms for these orders is shown in the bottom sub-panel.

\section{Analysis}

Our analysis of the data relies on the cross-correlation of the spectra with absorption models. This method requires very high-resolution data, because the precision of the crosscorrelation relies on the ability to resolve individual lines. The precision also increases with the number of absorption lines, and therefore molecules (whose rotation-vibration transitions produce thousands of absorption lines) are good targets for this type of analysis.

\subsection{Atmospheric Models}

In order to search for the Doppler-shifted signal from the thousands of absorption lines of water, we used a model that was calculated specifically for $55 \mathrm{Cnc}$ e using molecular data from the high-temperature molecular spectroscopic database (HITEMP; Rothman et al. 2010), assuming Voigt line profiles, with a line wing cutoff of $50 \mathrm{~cm}^{-1}$. It was then temperature-broadened using the standard database parameters, and Rayleigh scattering was included. Opacities were calculated line-by-line on a grid of 50 atmospheric layers, from 5 down to $10^{-10}$ bar, assuming a uniform temperature of $1000 \mathrm{~K}$ for pressures below $0.01 \mathrm{bar}, 1500 \mathrm{~K}$ for pressures from 0.01 to $1.0 \mathrm{bar}$, and $2500 \mathrm{~K}$ for pressures about $1.0 \mathrm{bar}$. This temperature-pressure profile agrees with that used by Demory et al. (2016b). However, we note that the spectral shape is not a strong function of temperature. Parameters used in these calculations are the stellar radius $\left(0.943 R_{\odot}\right)$, 
Table 2

Stellar and Planetary Parameters Used in Analysis

\begin{tabular}{lll}
\hline \hline Parameter & Value & Reference \\
\hline Systemic velocity $\left(\mathrm{km} \mathrm{s}^{-1}\right)$ & 27.58 & Nidever et al. (2002) \\
Orbital Period (days) & $0.736542(3)$ & Dragomir et al. (2014) \\
Ephemeris (JD) & $2455962.067(2)$ & Dragomir et al. (2014) \\
Semimajor axis (au) & $0.01545(3)$ & Dragomir et al. (2014) \\
$R_{p} / R_{\star}$ & $0.019(8)$ & Dragomir et al. (2014) \\
$a / R_{\star}$ & $3.52(4)$ & Dragomir et al. (2014) \\
\hline
\end{tabular}

planetary radius $\left(2.1 R_{\oplus}\right)$, and surface gravity at the bottom atmospheric layer $\left(17.3 \mathrm{~m} \mathrm{~s}^{-2}\right.$ derived using a planet mass of $\left.7.8 M_{\oplus}\right)$. Opacities were integrated along slant paths from the direction of the star to the observer. Integration over altitude and location on the disc then yielded the transmission of the planet for a given wavelength.

Models were calculated for a grid of atmospheric volume mixing ratios $(\mathrm{VMR}=10 \%, 1 \%, 0.1 \%$, and $0.01 \%$ ) and mean molecular weights $\left(\mu=2,5,10,15 \mathrm{~g} \mathrm{~mol}^{-1}\right)$, over a wavelength range of 5000-8000 $\AA$ with 60,000 evenly spaced data points. See Appendix Figure 8 for an example plot of one of the models used in this analysis. In general, using a lower volume mixing ratio significantly reduces the strength of absorption features at the blue end of our spectral data, due to masking by Rayleigh scattering, whereas increasing the weight of the atmosphere scales down the strength of the lines equally for all wavelengths. Before use, all models were convolved to the resolution of the data they were used with.

\subsection{Cross-correlation}

For each night, each spectrum is correlated with models at Doppler shifts spanning $-250 \mathrm{~km} \mathrm{~s}^{-1}$ to $+250 \mathrm{~km} \mathrm{~s}^{-1}$ in steps of $1 \mathrm{~km} \mathrm{~s}^{-1}$. We then phase fold the correlation signal from the in-transit frames. To do this, we first shift each correlation to the reference frame of $55 \mathrm{Cnc} e$, in order to correct for the Earth's rotation and orbital motion, the star's systemic velocity, and the planet's radial velocity (see Table 2). The correlations are then interpolated to a common velocity grid, using a cubic spline. In-transit frames were selected using transit models calculated via Mandel \& Agol (2002) and parameters in Table 2. Each point in velocity space is then summed, resulting in a measurement of model-correlation strength as a function of systemic velocity for the in-transit data, where we expect to see the signal from the planet at $V_{\mathrm{sys}}=0 \mathrm{~km} \mathrm{~s}^{-1}$.

\subsection{Model Recovery Tests and Estimation of the Detection Significance}

In order to assess our ability to detect and constrain the properties of the atmosphere of $55 \mathrm{Cnc}$, we ran several injection/recovery tests. To inject a model, we multiplied each in-transit spectrum by an atmospheric model in the exoplanet's reference frame. This was done after aligning the spectra onto a common wavelength grid, but before any further steps. The data with the models injected were processed and analyzed in an identical way to the normal science data. By injecting a water signal of varying strength into the data, and determining which can be recovered by our analysis, were able to place sensitivity limits on our results.

Assessing the detection significance is important. We therefore randomly drew 28 corrected spectra from all the corrected spectra during a night, assigned an in-transit phase to each of the spectra, and subsequently correlated and phasefolded the selected spectra. We repeated this procedure 10,000 times in order to ascertain the $1 \sigma$ and $3 \sigma$ confidence intervals. To determine the significance of our detections, we compared these confidence intervals to the difference in correlation strength at $V_{\text {sys }}=0 \mathrm{~km} \mathrm{~s}^{-1}$ of the normal and injected data.

We also reran our injection/recovery test to assess the effect of interpolating the data to a common wavelength grid after injecting the model. In these tests, we used the same procedure, but instead injected the water model (with a mean molecular mass of $10 \mathrm{~g} \mathrm{~mol}^{-1}$ and a water VMR of $10 \%$ ) before interpolating to a common wavelength grid. A correlation of the data with the model injected before and after is shown in Appendix Figure 9. The differences between these correlations are not significant in comparison to the noise, which gives us confidence that the wavelength interpolation is not affecting our final results.

\section{Results and Discussion}

The correlation of our original and injected data can be found in Figure 1, along with a plot of the correlation after phase folding to $55 \mathrm{Cnc}$ e's orbital velocity. The model injected in Figure 1 utilizes a high water VMR of $10 \%$ and a low mean molecular weight of $2 \mathrm{~g} \mathrm{~mol}^{-1}$; it can easily be seen in the injected correlation as a dark diagonal trace in the in-transit frames. The slope of the trace corresponds to the planet's radial velocity change during the transit, with respect to the systemic velocity. We again note that our reduction was not able to remove all contamination from tellurics. Residuals can be seen in the out-of-transit correlations in the left and center panels of Figure 1. The features at phases $0.05-0.2$ and $\sim 60 \mathrm{~km} \mathrm{~s}^{-1}$ are telluric residuals from Night 2, where the combined heliocentric and systemic velocity is $\sim 60 \mathrm{~km} \mathrm{~s}^{-1}$. However, because the correlations used in our final analysis are only from the in-transit frames, this contamination should not influence our results.

In the phase-folded plot in the right panel of Figure 1, the injected signal is detected at $>20 \sigma$ at $V_{\text {sys }}=0$. However, the original data exhibits no significant features in both the correlated and phase-folded data.

Although there is no significant $(>3 \sigma)$ peak in our data, there are many peaks at more than $1 \sigma$. Because the many large features could hide the signal from the planet, we want to understand their cause. As the spectrum from water is very closely spaced, it is likely that noise spikes are picked up by adjacent water lines. To test this, we simulated a data set with purely white noise at a signal-to-noise ratio of 300 . We correlated and phase-folded these simulated spectra (both with and without a planetary signal injected). We also used the same analysis to assess the significance of any features. The results from this analysis can be seen in Figure 2. It is clear that the white noise gives rise to a similar structure.

Although Figure 1 demonstrates that we can clearly rule out a large scale-height, very water-rich model, it does not allow us to immediately constrain the composition and scale-height of 

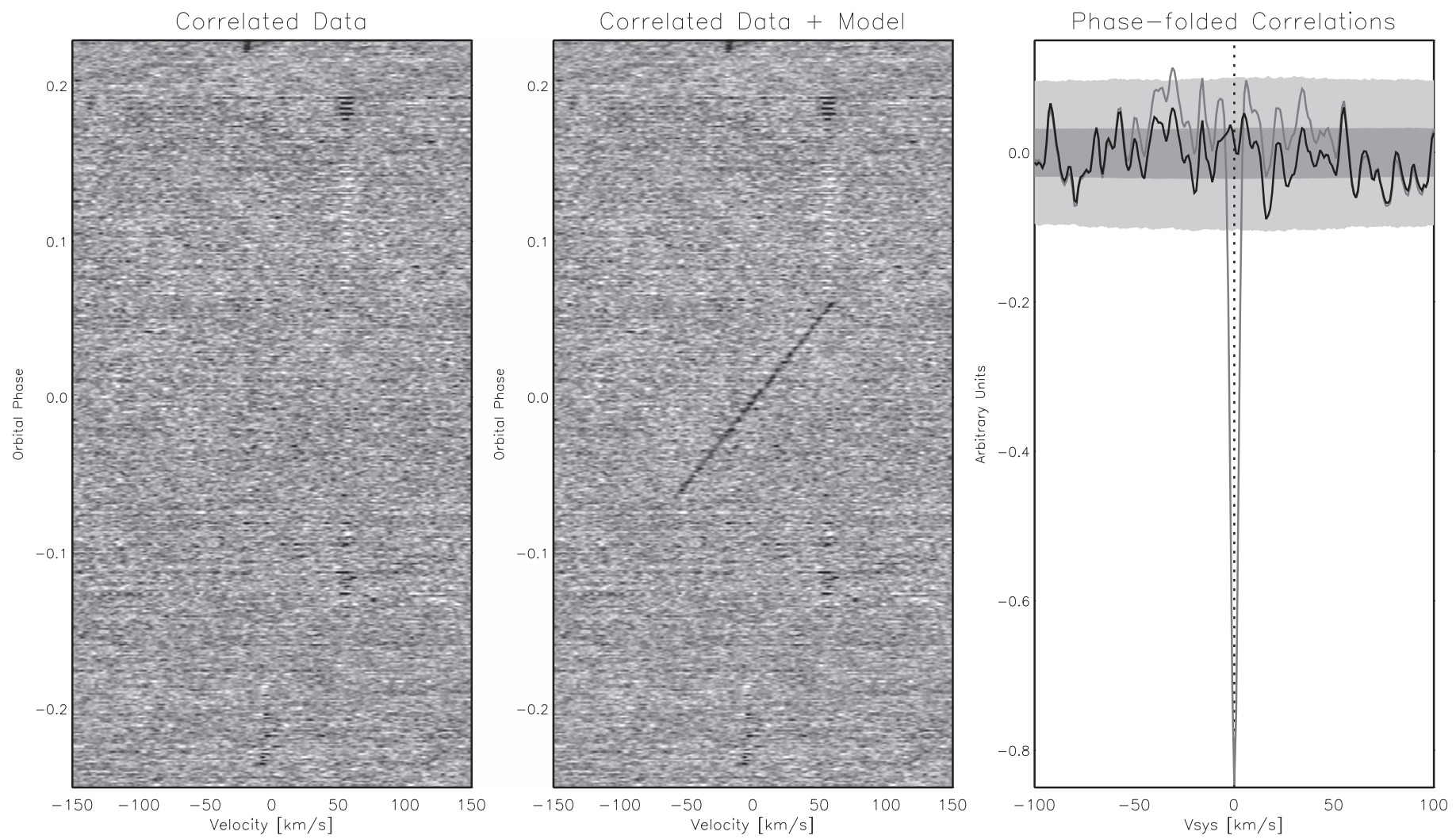

Figure 1. Left panel: correlated data from all four nights of observations. Middle panel: correlated data + model with a water VMR of $10 \%$ and a low mean molecular weight of $2 \mathrm{~g} \mathrm{~mol}^{-1}$. Right panel: correlation phase-folded to the planet's orbital radial velocity. The black line is the data and the gray line is the data + model. The dark gray region represents $1 \sigma$ values for each data point, whereas the light gray region represents the $3 \sigma$ values. For a description of how these were calculated, see Section 4.3.
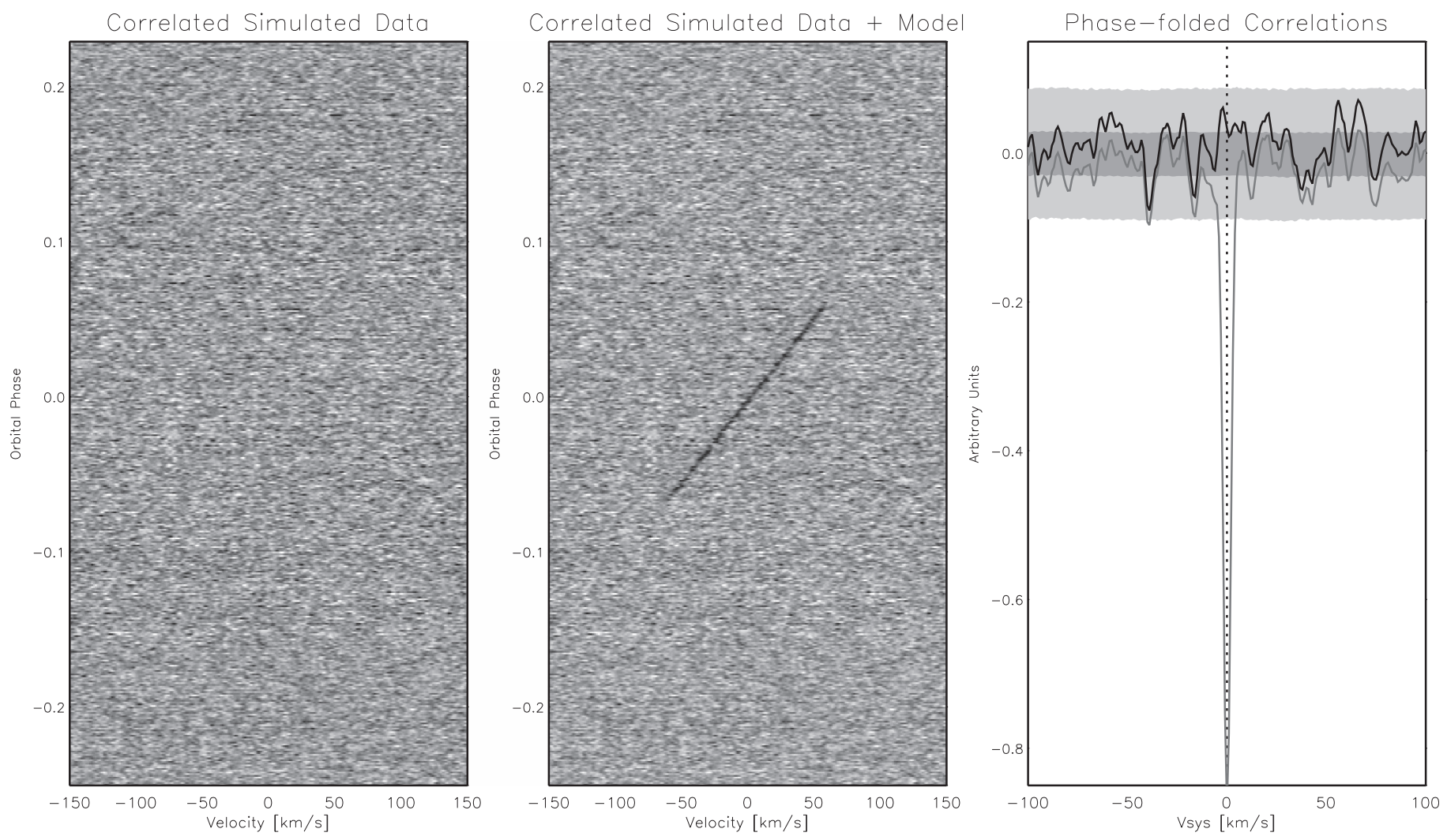

Figure 2. Correlated and phase-folded simulated data, with normally distributed noise and a signal-to-noise ratio of 300. For a description of each panel see Figure 1. 


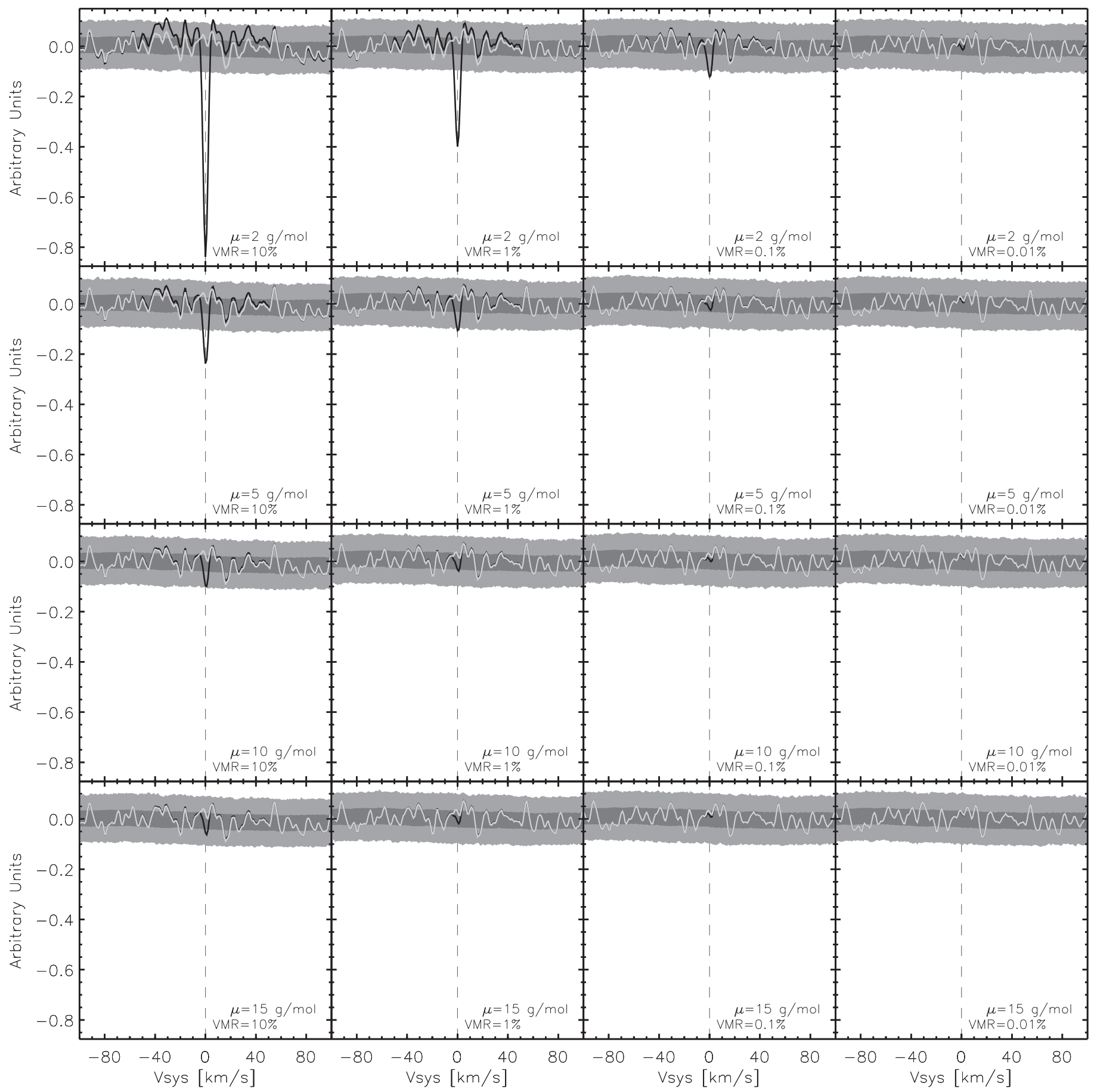

Figure 3. Phase-folded correlations for a range of models that vary in volume-mixing ratio and mean molecular mass. The black line shows the data and the gray line shows the data + model. The dark gray region represents $1 \sigma$ values for each data point, whereas the light gray region represents the $3 \sigma$ values.

55Cnc e's atmosphere. We therefore inject models at different VMR and $\mu$ to see which model we would be able to detect at $3 \sigma$. The phase-folded correlations are shown in Figure 3.

By comparing the phase-folded correlations, normal and injected, we are able to place a $3 \sigma$ lower limit of $10 \mathrm{~g} \mathrm{~mol}^{-1}$ on the mean-molecular weight of $55 \mathrm{Cnc}$ e's water-rich (volume mixing ratio $>10 \%$ ), optically thin atmosphere, which corresponds to an atmospheric scale-height of $\sim 80 \mathrm{~km}$. This implies that, if $55 \mathrm{Cnc} \mathrm{e}$ has a water-rich atmosphere, as suggested by Gillon et al. (2012) and Winn et al. (2011), and is optically thin, it must have a mean molecular weight heavier than $10 \mathrm{~g} \mathrm{~mol}^{-1}$. The phase-folded correlations of the waterdepleted atmosphere models, $1 \%$ and $0.1 \%$ water by volume, yielded $3 \sigma$ lower limits of 5 and $2 \mathrm{~g} \mathrm{~mol}^{-1}$, respectively, but we could not detect a signal from a $0.01 \%$ water atmosphere. Our result, that $55 \mathrm{Cnc}$ e does not have a water-rich, optically thin extended atmosphere, is not surprising because water, with its mean molecular mass of $18 \mathrm{~g} \mathrm{~mol}^{-1}$, would produce a heavy, and therefore compact, atmosphere if it were a large fraction by mass. 
We have shown that, if $55 \mathrm{Cnc}$ e has an optically thin extended atmosphere, it is not water-rich. Evidence for the existence of an extended atmosphere on the planet includes detections reported by Tsiaras et al. (2016) of features at 1.42 and $1.54 \mu \mathrm{m}$, which they attribute to trace amounts of HCN retained within the planet's extended atmosphere. Additional evidence includes the tentative detection of sodium and singly ionized calcium reported by Ridden-Harper et al. (2016), as well as findings from Lammer et al. (2013) that 55Cnc e's theoretical mass-loss rate is $\sim 10$ times lower than that of a typical hot-Jupiter, and therefore $55 \mathrm{Cnc}$ e can retain a hydrogen envelope. Furthermore, a water-depleted atmosphere was predicted by Ito et al. (2015), who modeled an atmosphere that is in gas/melt equilibrium with an underlying magma ocean; a possible scenario for $55 \mathrm{Cnc}$ e, given its high temperature. They found that their equilibrium calculations yielded a water-depleted mineral atmosphere (i.e., $\mathrm{Na}, \mathrm{K}, \mathrm{Fe}$, $\mathrm{Si}, \mathrm{SiO}, \mathrm{O}$, and $\mathrm{O}_{2}$ as the major atmospheric species).

We note that, although the opacity of 55Cnc e's atmosphere is not known, our mean molecular mass limits assume that the planet's atmosphere is optically thin (i.e., the signal is not being masked by clouds or hazes). Indirect evidence that $55 \mathrm{Cnc} \mathrm{e}$ possibly harbors an optically thick atmosphere was presented by Demory et al. (2016a, 2016b) and corroborated by Kite et al. (2016). The latter study finds that heat transport by magma cannot explain the antistellar-hemisphere temperature found by Demory et al. (2016b) and concludes that an optically thick atmosphere is likely required to transport heat to the planet's night-side.

We conclude that our results are compatible with a cloudy atmosphere, as well as an optically thin one. In the cloudy scenario, we cannot place any constraints on the planet's weight or composition, due to the fact that absorption features can be partially or fully obscured by clouds and other aerosols. In the optically thin scenario, our result is compatible with a lightweight atmosphere that is depleted of water (VMR $<10 \%)$, as well as a heavy atmosphere with any composition, including water-rich.

Although our analysis did not detect water on $55 \mathrm{Cnc}$ e, our injection and recovery tests demonstrate that it is possible to recover known water-vapor absorption signals in a nearby superEarth atmosphere, using high-resolution transit spectroscopy with current ground-based instruments. At the moment, only a couple of nearby super-Earth are known. However, upcoming surveys and missions, such as TESS, are expected to reveal many terrestrial planets, some of which will be excellent targets for follow-up observations of their atmospheres using the highresolution Doppler cross-correlation technique.

This work was based in part on data collected on HDS at the Subaru Telescope, which is operated by the National Astronomical Observatory of Japan, and on data collected by ESPaDOnS at the Canada-Hawaii-France Telescope, which is operated by the National Research Council (NRC) of Canada, the Institut National des Science de l'Univers of the Centre National de la Recherche Scientifique (CNRS) of France, and the University of Hawaii. This work was supported by grants to R.J. from the Natural Sciences and Engineering Research Council (NSERC) of Canada. L.J.E. is supported in part by an NSERC CGS, E.J.W.M. was funded by the Michael West Fellowship, and C.A.W. acknowledges support by STFC grant ST/L000709/1.

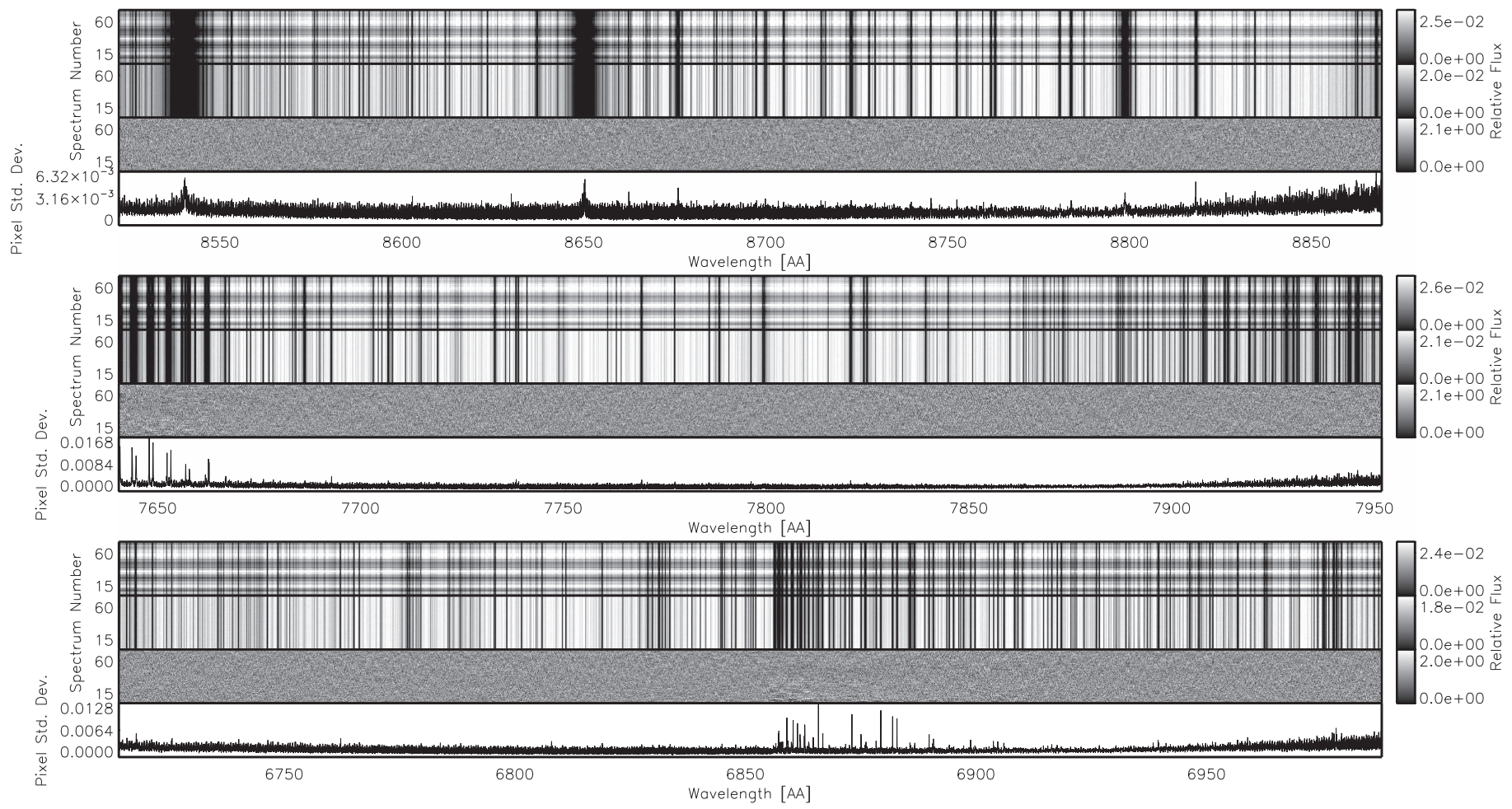

Figure 4. Top three panels: stages of our reduction for a sample order of data from the first night of CFHT observations ( $\mathrm{N}_{1}$ ). First sub-panel from the top: the raw 1D spectra (see Section 3.1). Second sub-panel from the top: after normalization (see Section 3.2). Third sub-panel from the top: after stellar and telluric line removal (see Section 3.2). Fourth sub-panel from the top: the standard deviation of each data point in the reduced spectra. 


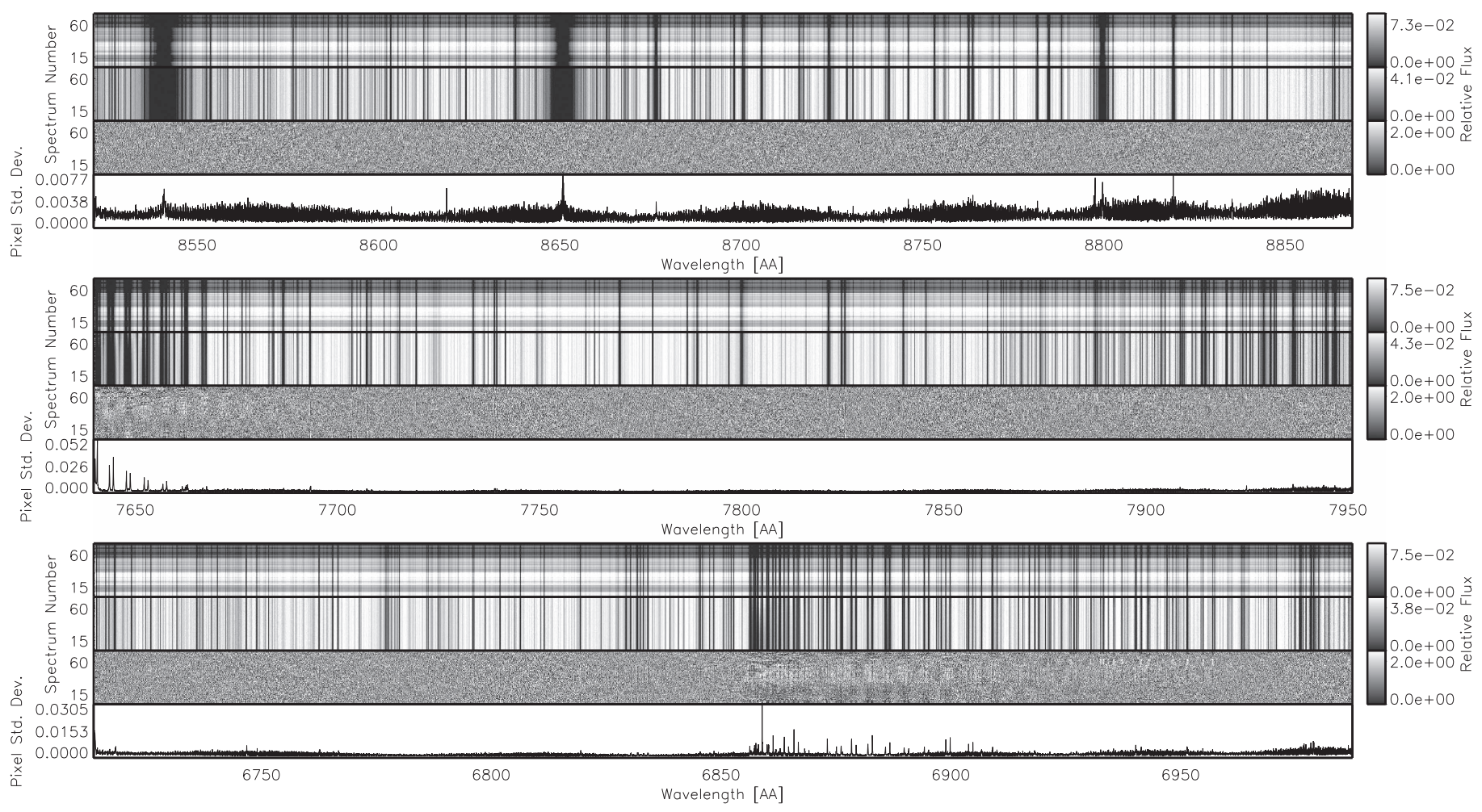

Figure 5. Each panel: stages of our reduction for a sample order of data from the second night of CFHT observations $\left(\mathrm{N}_{2}\right)$. For a description of each sub-panel, see Figure 4.

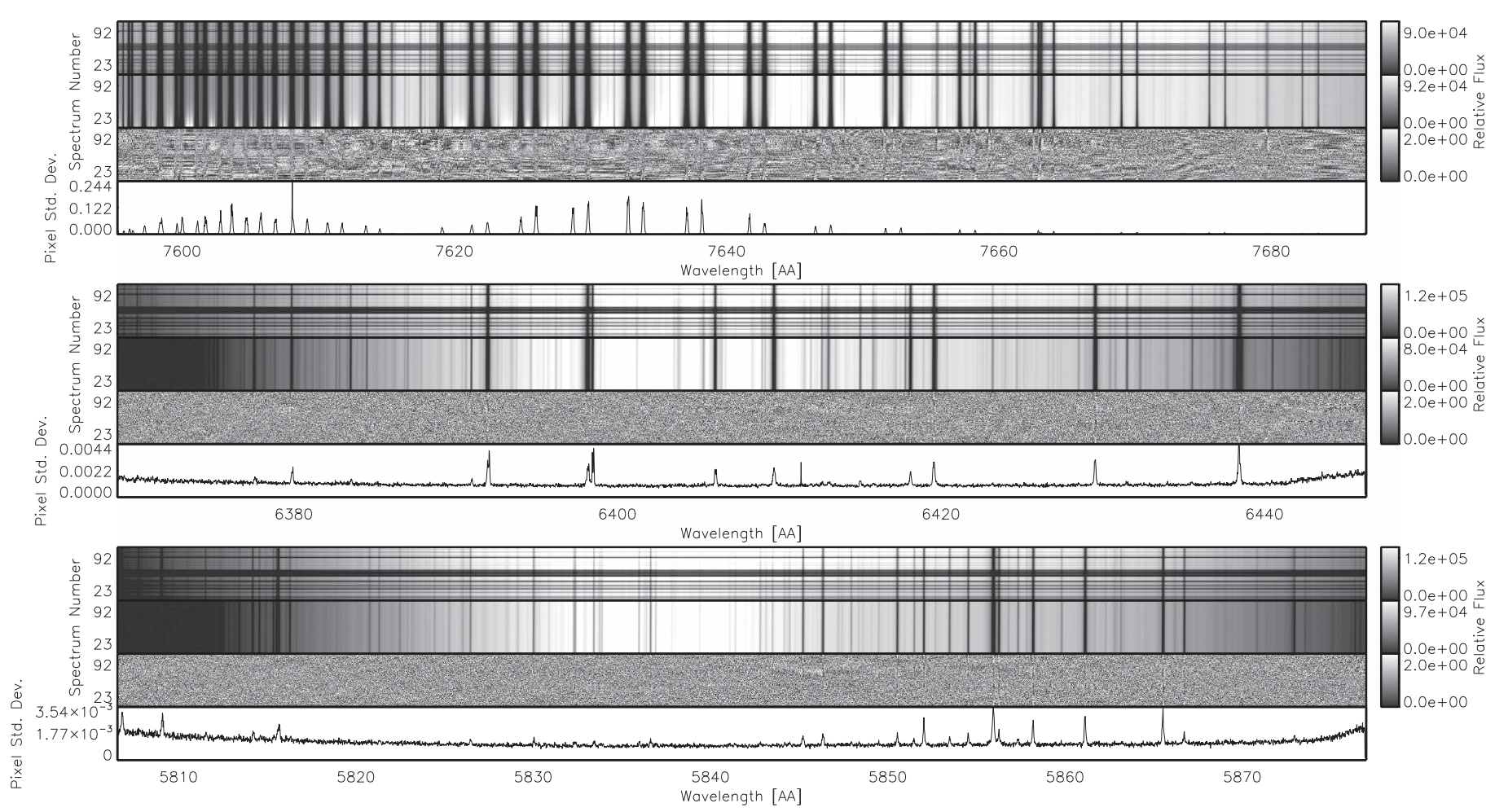

Figure 6. Each panel: stages of our reduction for a sample order of data from the first night of Subaru observations $\left(\mathrm{N}_{3}\right)$. For a description of each sub-panel, see Figure 4. 

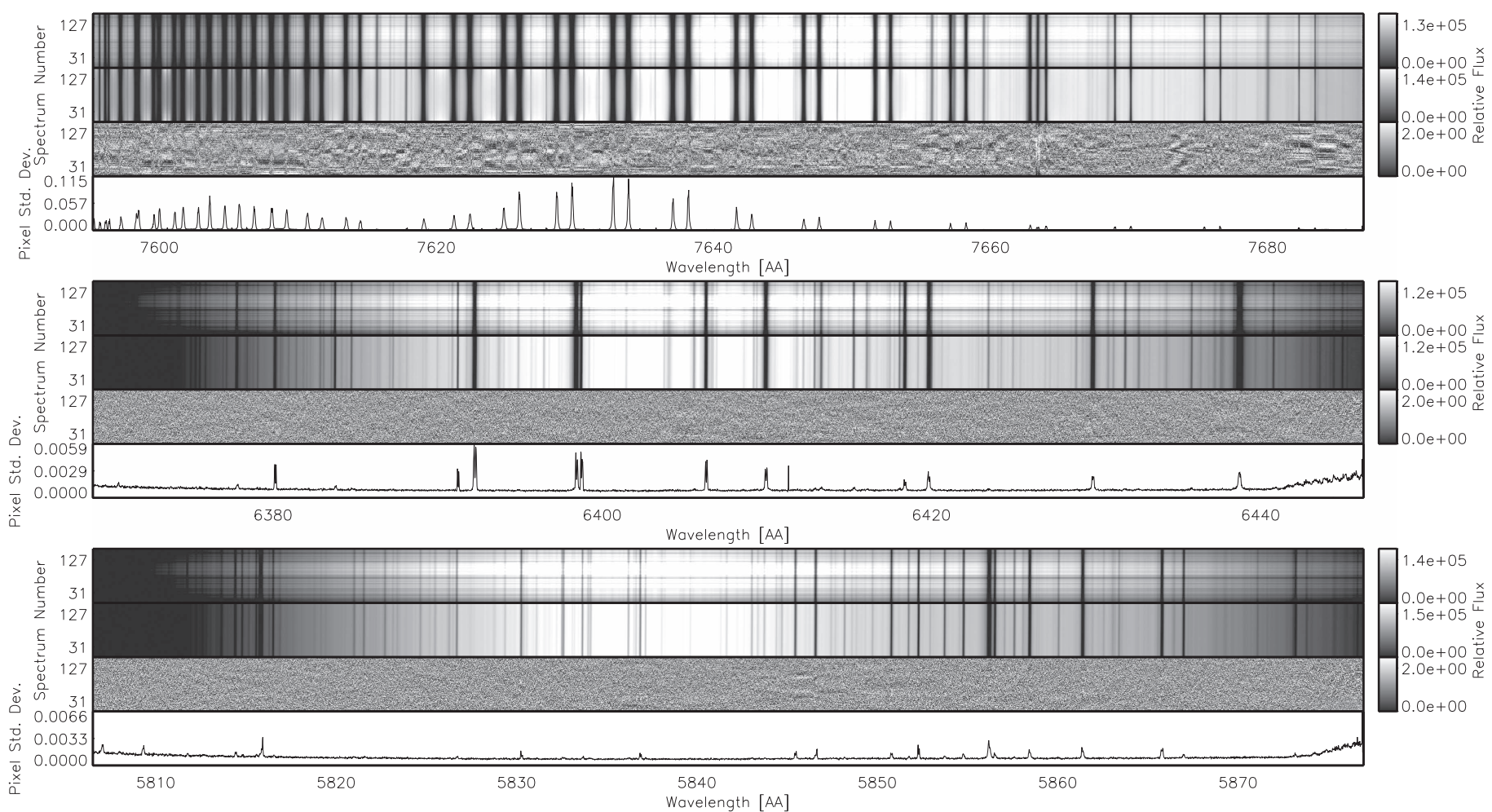

Figure 7. Each panel: stages of our reduction for a sample order of data from the second night of Subaru observations $\left(\mathrm{N}_{4}\right)$. For a description of each sub-panel, see Figure 4.
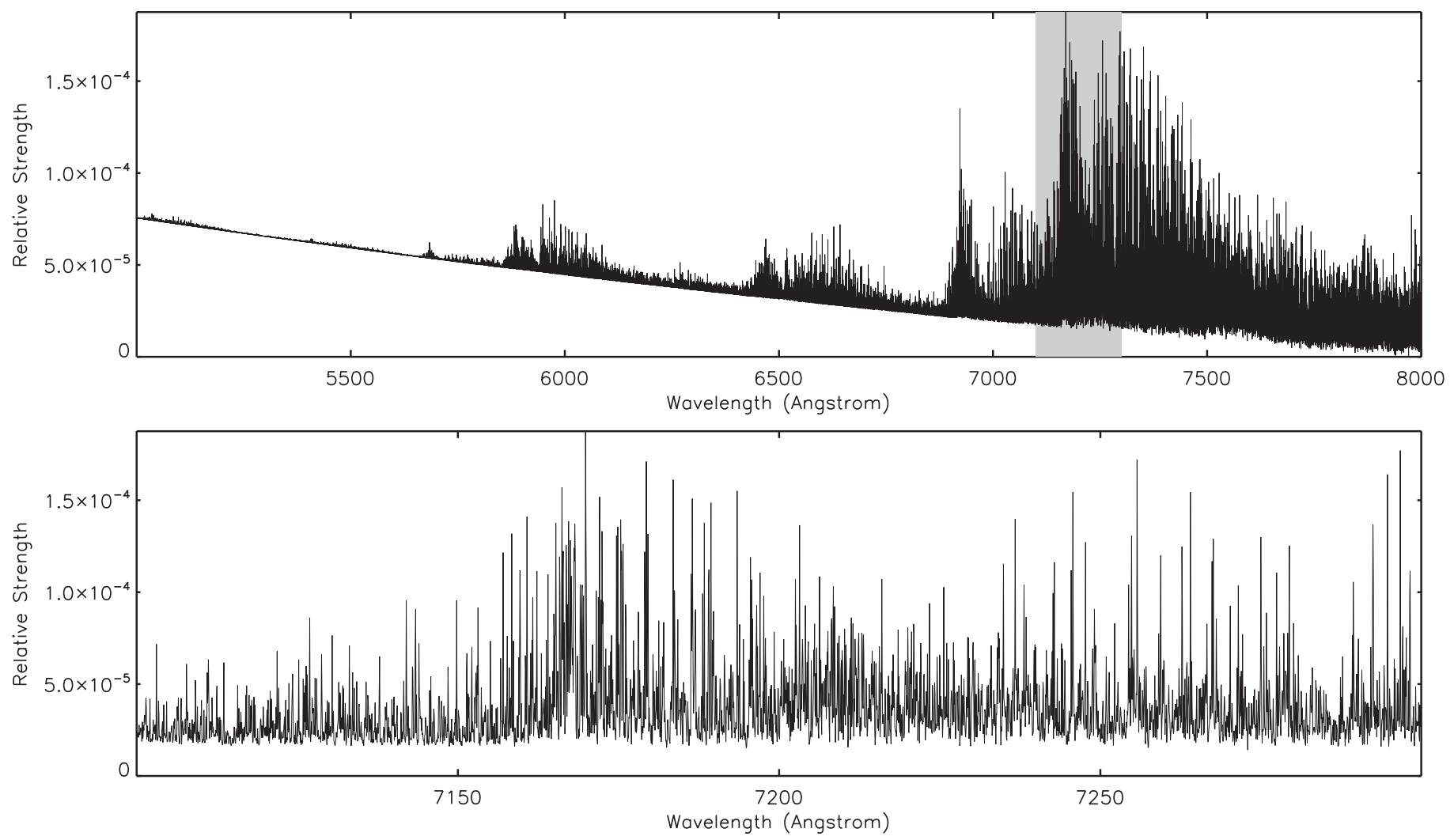

Figure 8. Top panel: an example of a model spectrum for water absorption used in this analysis. The model corresponding to a water-rich atmosphere with a mean molecular mass of $10 \mathrm{~g} \mathrm{~mol}^{-1}$ and a water volume mixing ratio of $10 \%$. Bottom panel: a close-up of the gray region of the spectra presented in the top panel. 


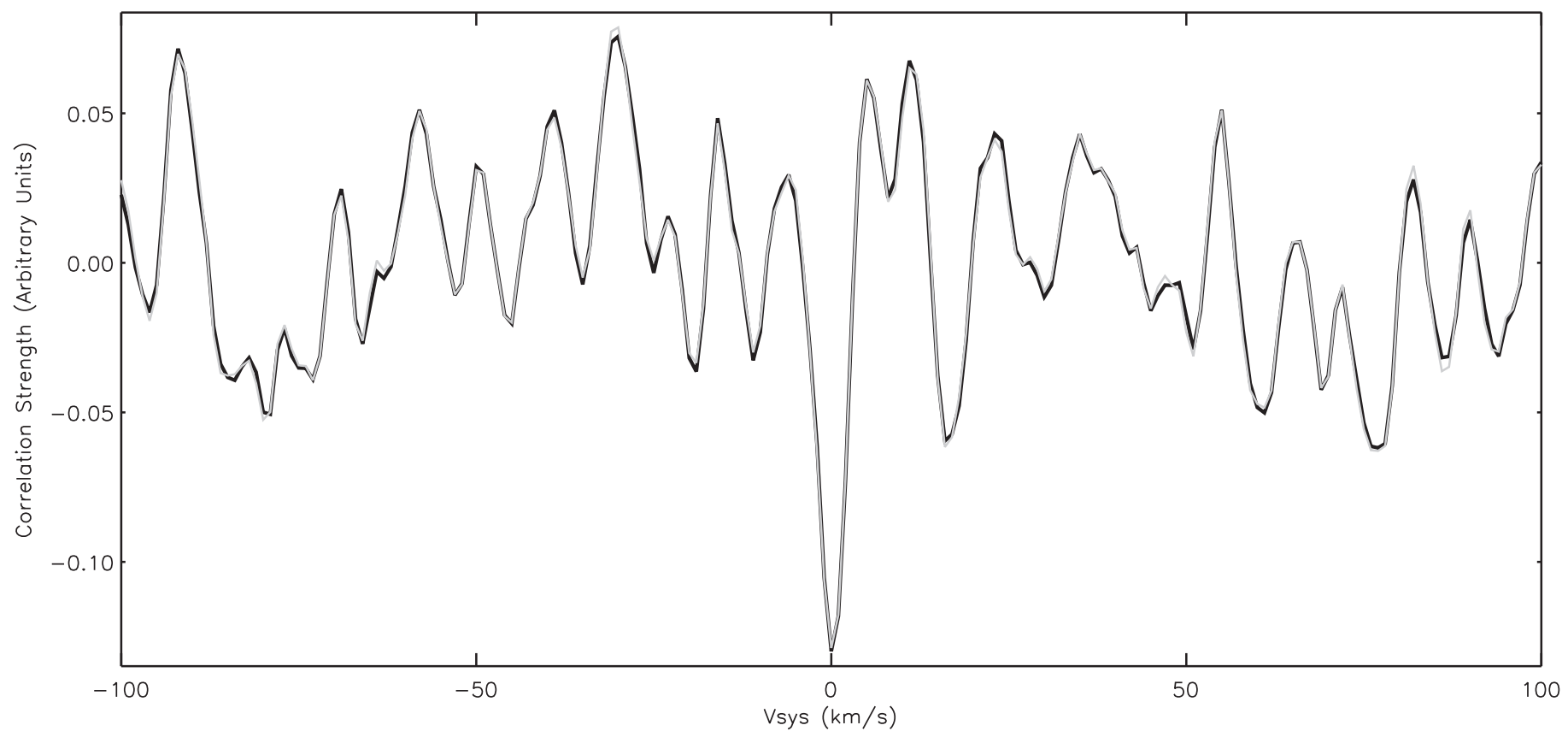

Figure 9. Correlation strength of the water-model injected data, as a function of systemic velocity. The water model used has a mean molecular mass of $10 \mathrm{~g}$ mol ${ }^{-1}$ and a water VMR of $10 \%$. In black is the correlation of data with the model injected before interpolating to a common wavelength grid, whereas gray is the correlation with the model injected after the interpolation.

\section{References}

Bean, J. L., Désert, J.-M., Kabath, P., et al. 2011, ApJ, 743, 92 Bean, J. L., Miller-Ricci Kempton, E., \& Homeier, D. 2010, Natur, 468, 669 Birkby, J. L., de Kok, R. J., Brogi, M., et al. 2013, MNRAS, 436, L35 Brogi, M., de Kok, R. J., Albrecht, S., et al. 2016, ApJ, 817, 106 Brogi, M., de Kok, R. J., Birkby, J. L., Schwarz, H., \& Snellen, I. A. G. 2014 , A\&A, 565, A124

Brogi, M., Snellen, I. A. G., de Kok, R. J., et al. 2012, Natur, 486, 502 Brogi, M., Snellen, I. A. G., de Kok, R. J., et al. 2013, ApJ, 767, 27 Charbonneau, D., Allen, L. E., Megeath, S. T., et al. 2005, ApJ, 626, 523 Charbonneau, D., Berta, Z. K., Irwin, J., et al. 2009, Natur, 462, 891

Charbonneau, D., Brown, T. M., Noyes, R. W., \& Gilliland, R. L. 2002, ApJ, 568,377

Dawson, R. I., \& Fabrycky, D. C. 2010, ApJ, 722, 937

de Kok, R. J., Brogi, M., Snellen, I. A. G., et al. 2013, A\&A, 554, A82

de Mooij, E. J. W., López-Morales, M., Karjalainen, R., Hrudkova, M., \& Jayawardhana, R. 2014, ApJL, 797, L21

de Mooij, E. J. W., \& Snellen, I. A. G. 2009, A\&A, 493, L35

Delgado Mena, E., Israelian, G., González Hernández, J. I., et al. 2010, ApJ, 725,2349

Deming, D., Seager, S., Richardson, L. J., \& Harrington, J. 2005, Natur, 434, 740

Deming, D., Wilkins, A., McCullough, P., et al. 2013, ApJ, 774, 95

Demory, B.-O., Gillon, M., de Wit, J., et al. 2016a, Natur, 532, 207

Demory, B.-O., Gillon, M., Deming, D., et al. 2011, A\&A, 533, A114

Demory, B.-O., Gillon, M., Madhusudhan, N., \& Queloz, D. 2016b, MNRAS, 455, 2018

Demory, B.-O., Gillon, M., Seager, S., et al. 2012, ApJL, 751, L28

Donati, J.-F. 2003, in ASP Conf. Ser. 307, Solar Polarization, ed. J. Trujillo-Bueno \& J. S. Almeida (San Francisco, CA: ASP), 41

Donati, J.-F., Semel, M., Carter, B. D., Rees, D. E., \& Collier Cameron, A. 1997, MNRAS, 291, 658

Dragomir, D., Matthews, J. M., Winn, J. N., \& Rowe, J. F. 2014, in IAU Symp. 293, Formation, Detection, and Characterization of Extrasolar Habitable Planets, ed. N. Haghighipour (Cambridge: Cambridge Univ. Press), 52

Ehrenreich, D., Bourrier, V., Bonfils, X., et al. 2012, A\&A, 547, A18
Fischer, D. A., Marcy, G. W., Butler, R. P., et al. 2008, ApJ, 675, 790 Gillon, M., Demory, B.-O., Benneke, B., et al. 2012, A\&A, 539, A28 Hu, R., \& Seager, S. 2014, ApJ, 784, 63

Ito, Y., Ikoma, M., Kawahara, H., et al. 2015, ApJ, 801, 144 Kite, E. S., Fegley, B., Jr., Schaefer, L., \& Gaidos, E. 2016, ApJ, 828, 80

Knutson, H. A., Dragomir, D., Kreidberg, L., et al. 2014, ApJ, 794, 155 Kreidberg, L., Bean, J. L., Désert, J.-M., et al. 2014, Natur, 505, 69 Lammer, H., Erkaev, N. V., Odert, P., et al. 2013, MNRAS, 430, 1247 Lockwood, A. C., Johnson, J. A., Bender, C. F., et al. 2014, ApJL, 783, L29 Madhusudhan, N., Lee, K. K. M., \& Mousis, O. 2012, ApJL, 759, L40 Mandel, K., \& Agol, E. 2002, ApJL, 580, L171

Martins, J. H. C., Santos, N. C., Figueira, P., et al. 2015, A\&A, 576, A134

McArthur, B. E., Endl, M., Cochran, W. D., et al. 2004, ApJL, 614, L81

Nidever, D. L., Marcy, G. W., Butler, R. P., Fischer, D. A., \& Vogt, S. S. 2002, ApJS, 141, 503

Noguchi, K., Aoki, W., Kawanomoto, S., et al. 2002, PASJ, 54, 855

Ridden-Harper, A. R., Snellen, I. A. G., Keller, C. U., et al. 2016, A\&A, 593, A 129

Rodler, F., Kürster, M., \& Barnes, J. R. 2013a, MNRAS, 432, 1980

Rodler, F., Kürster, M., López-Morales, M., \& Ribas, I. 2013b, AN, 334, 188

Rodler, F., Lopez-Morales, M., \& Ribas, I. 2012, ApJL, 753, L25

Rothman, L. S., Gordon, I. E., Barber, R. J., et al. 2010, JQSRT, 111, 2139

Sing, D. K., Fortney, J. J., Nikolov, N., et al. 2016, Natur, 529, 59

Sing, D. K., \& López-Morales, M. 2009, A\&A, 493, L31

Snellen, I. A. G., Brandl, B. R., de Kok, R. J., et al. 2014, Natur, 509, 63

Snellen, I. A. G., de Kok, R. J., de Mooij, E. J. W., \& Albrecht, S. 2010, Natur, 465,1049

Stevenson, K. B., Désert, J.-M., Line, M. R., et al. 2014, Sci, 346, 838

Swain, M. R., Tinetti, G., Vasisht, G., et al. 2009, ApJ, 704, 1616

Tajitsu, A., Aoki, W., \& Yamamuro, T. 2012, PASJ, 64, 77

Tamuz, O., Mazeh, T., \& Zucker, S. 2005, MNRAS, 356, 1466

Teske, J. K., Cunha, K., Schuler, S. C., Griffith, C. A., \& Smith, V. V. 2013, ApJ, 778, 132

Tsiaras, A., Rocchetto, M., Waldmann, I. P., et al. 2016, ApJ, 820, 99

Valencia, D., Ikoma, M., Guillot, T., \& Nettelmann, N. 2010, A\&A, 516 A20

Winn, J. N., Matthews, J. M., Dawson, R. I., et al. 2011, ApJL, 737, L18 